\title{
Los jóvenes de la Universidad Católica Silva Henríquez (UCSH) como sujetos políticos en los movimientos sociales ${ }^{1}$
}

\author{
Sonia Brito Rodríguez ${ }^{2}$ \\ Margarita Posada Lecompte ${ }^{3}$ \\ Nicolás Castro Solís ${ }^{4}$
}

\section{RESUMEN}

El presente artículo se refiere a la comprensión de sí mismos que tienen los estudiantes de la UCSH en tanto sujetos políticos y sus prácticas ciudadanas y democráticas, en relación con los movimientos estudiantiles de educación media y superior de los últimos 10 años. En 2017 se realizó una investigación mediante la estrategia de grupos de discusión y entrevistas en profundidad, en la cual participaron 38 informantes clave (estudiantes de las diversas facultades y egresados de la UCSH) que han sido delegados de sus carreras o participan en la federación de estudiantes. Del análisis de las narraciones se desprende que los jóvenes se reconocen como agentes políticos capaces de movilizar personas, ideas y proyectos de cambio en torno a la educación y a la transformación del país. Ellos comprenden y actúan en lo político, denunciando y demandando cambios estructurales; para ello utilizan estrategias que emplazan a la institución y a la sociedad civil a visibilizar una problemática postergada y la instalan en la agenda pública. Estos movimientos juveniles promueven una nueva forma de entender la política, la participación, y de ejercer la ciudadanía en la búsqueda de mayores condiciones de equidad y justicia social.

Palabras clave: Jóvenes, educación superior, movimientos estudiantiles, democracia, sujetos políticos

1 Artículo recibido 20/09/2018. Artículo aprobado 06/12/2018.

2 Chilena, Trabajadora Social, Académica- investigadora. Universidad Católica Silva Henríquez, Santiago, Chile.E-mail: sbritor@ucsh.cl.

3 Colombiana, Psicóloga, Docente investigadora adjunta, Universidad Católica Silva Henríquez, Santiago, Chile.E-mail: mposadal@ucsh.cl

4 Chileno, Trabajador Social, ayudante de investigación. E-mail: ncastros@miucsh.cl 


\title{
Young people in Universidad Católica Silva Henríquez (UCSH) as political subjects in social movements
}

\begin{abstract}
This article refers to the UCSH students' self-perception as political subjects and to their citizen and democratic practices in relation to the secondary and higher education students' movements from the last 10 years on. In 2017, the research carried out through the strategy of discussion groups and indepth interviews worked with 38 key informants (students and graduates from various UCSH faculties) who had been delegates from their careers or participated in the student federation. From the analysis of narrations, it is clear that young people consider themselves as political agents capable of mobilizing people, ideas and projects of change around education and transformation of the country. They have understanding and action in the political area, denouncing and demanding structural changes and to do so, they use strategies that force institutions and civil society to visualize an omitted problem and include it in the public agenda. These youth movements promote a new way of understanding politics, participation and exercising citizenship for enhancing conditions of equity and social justice.
\end{abstract}

Keywords: Young people, higher education, student movements, democracy, political subjects

\section{Os jovens da Universidade Católica Silva Henríquez (UCSH) como sujeitos políticos nos movimentos sociais}

\section{RESUMO}

O presente artigo refere à compreensão de si mesmos que têm os estudantes da UCSH, quanto sujeitos políticos e suas práticas cidadãs e democráticas, em relação com os movimentos estudantis de educação secundária e superior dos últimos 10 anos. No ano 2017 foi realizada uma pesquisa através da estratégia de grupos de discussão e entrevistas em profundidade, na qual participaram 38 informantes-chave (estudantes das diversas faculdades e formados pela UCSH) que têm sido representantes de suas carreiras ou participam na federação de estudantes. Da análise das narrações conclui-se que os jovens se reconhecem como agentes políticos capazes de mobilizarem pessoas, ideias e projetos de mudança em torno da educação e a transformação do país. Eles compreendem e agem no político, denunciando e demandando mudanças estruturais, para aquilo, utilizam estratégias que chamam à instituição e à sociedade civil a visibilizarem uma problemática adiada e a instalam na agenda pública. Estes movimentos juvenis promovem uma nova forma de entender a política, a participação e exercer a cidadania na busca de maiores condições de equidade e justiça social.

Palavras-chave: Jovens, educação superior, movimentos estudantis, democracia, sujeitos políticos 


\section{Introducción}

El documento recoge las principales narrativas de los estudiantes y egresados que han sido parte de los movimientos juveniles en la UCSH, quienes han compartido en los grupos de discusión sus percepciones y prácticas de participación, ciudadanía, política y democracia en torno a las preguntas: ¿cuáles son las comprensiones que tienen de sí mismos como sujetos políticos y su influencia en las acciones de los movimientos estudiantiles de la UCSH?, y ¿cuáles son las prácticas de ejercicio ciudadano y político que llevan a cabo?

El objetivo de la investigación fue "Describir las percepciones, comprensiones, compromiso y prácticas políticas de jóvenes universitarios de la UCSH, participes de los movimientos estudiantiles de los últimos 10 años, tanto a nivel de educación media como superior, a fin de analizar su valoración y significancia de estos en la construcción de país".

La investigación se realiza desde la psicología de lo popular que, según Muñoz (2002), rescata las descripciones "que hacen las personas acerca de cómo funcionan los seres humanos, cómo son las mentes propias y de los demás, situada en los contextos cotidianos en los que vive la gente (...) Su principio organizativo es narrativo" (p. 2). Por tanto, la narrativa está basada en lo que la gente hace, o dice que hace, así como las razones de su actuar en el mundo. Por ello, en esta investigación se recogen las narrativas que los estudiantes y egresados de la UCSH tienen acerca de sí mismos y de los otros en relación con los movimientos estudiantiles y, con ello, con los fenómenos como el paro, la toma, la apropiación territorial, la asamblea, y que ellos expresan a partir de sus vivencias.

Adoptar esta aproximación metodológica en esta investigación, desde Holstein y Gubrium (2008) (citados por Sancho, Hernández, Herraiz \& Vidiella (2009, p. 1159), implica "asumir que la realidad social y las representaciones que la median, así como los discursos que la fijan y posicionan a los sujetos son construidos 
socialmente mediante prácticas de significación". Por tanto, esta investigación se propone, como plantean Sancho et al. (2009),

tratar de comprender el mundo de los significados y clarificar qué y cómo están mediados tanto en el lenguaje como en las acciones de los actores sociales; sin olvidar que una interpretación es, en sí misma, la construcción de una lectura de esos significados. (p. 1159)

Por tanto, esta investigación busca ahondar en las comprensiones que los jóvenes han construido acerca de sus procesos, desde el paradigma interpretativo-hermenéutico, que alude a una investigación de alcance cualitativo-comprensiva. Esta elección permite acceder y aproximarse a los actores educativos desde la propia visión de los sujetos (estudiantes y egresados con experiencias en la dirigencia o representación de alguna carrera de la Universidad).

Por tanto, es relevante analizar los cambios culturales que han acontecido en la sociedad chilena, los cuales imponen un nuevo escenario de oportunidades y amenazas desde el que debe comprenderse la juventud. Dado que los jóvenes, según indica Weber (2002), "(incluyendo tolerancia u omisión) se orientan por las acciones de otros, las cuales pueden ser pasadas, presentes o esperadas como futuras". (p. 18). De este modo, están inspiradas en situaciones nacionales o foráneas, pero tienen una propia lectura de sí y de su forma de operar. Como aclara Weber (2002), esta "acción social no es idéntica: a) ni a una acción homogénea de muchos; b) ni a la acción de alguien influido por conductas de otros". (p. 19). En tal sentido, señala González (2012), "el surgimiento de modos alternativos de participación y disidencia precisan estructurar nuevas miradas para analizar los vínculos y las rupturas entre estos actores y la esfera pública" (p.148).

Tal como indican García- Cabrero, Loredo, Luna y Rueda (2008), dado que la educación superior es el espacio propicio para la formación de ciudadanos que aporten a la construcción de un mundo más justo, equitativo, inclusivo y sustentable, es necesario com- 
prender los fenómenos que en este entorno acontecen. Es decir, para emplear su potencial trasformador y formar jóvenes comprometidos con la sociedad, con realidades diversas y desde una perspectiva ética y ecológica, es necesario aproximarse a su visión de mundo.

Es por ello que las políticas educativas de un país democrático deben garantizar que los jóvenes tengan las suficientes oportunidades de educarse en un ambiente de calidad, tanto para su vida personal como profesional; eso incluye su formación como ciudadanos, capaces de actuar de manera libre y responsable en la construcción de una sociedad mejor. Asimismo, esa formación debe favorecer elegir libremente un tipo de trabajo u ocupación, una forma de vida, así como participar e influir para que los mecanismos sociales sean más efectivos. Lind (2003) señala que la educación de buena calidad forma en las habilidades y actitudes que el individuo requiere para hacer una carrera, así como también promueve competencias y posturas para mantener la paz y la democracia en un contexto complejo, antagonista y rápidamente cambiante.

Como señala Bendit (2000), las entidades educativas deben autoobservar y cuestionar sus prácticas y propuestas, toda vez que los jóvenes han cambiado su manera de comprender y valorar las estructuras, sus expectativas y el futuro. Como menciona Rodríguez (2013), es necesario entonces "escuchar más y mejor los mensajes que estos movimientos juveniles están formulando, y de generar diálogos más fluidos entre éstos y los operadores de políticas públicas de juventud, reformulando las estrategias con las que se ha trabajado hasta el momento" (p. 2). El reconocer estas voces, este cambio, implica ofrecer y consensuar formas de participar que se ajusten a las nuevas necesidades de los ciudadanos.

De esta manera, las instituciones de educación superior deben promover y gestar ideas y propuestas formativas que compensen las desigualdades e inequidades de los modelos socioeconómicos vigentes, buscando nuevas alternativas de desarrollo, que propi- 
cien la formación de un ciudadano activo y participativo. Al respecto Braslavsky (2006) indica que:

la educación le tiene que servir a las personas y a los grupos para operar en el mundo y para sentirse bien operando en ese mundo: conociéndolo, interpretándolo, transformándolo en una relación fértil y creativa entre sí y con el entorno. (p. 1)

Por tanto, para un país competitivo, la educación debe ser de calidad, equitativa y eficiente. Sin embargo, la experiencia de esta gestión política y social en Chile no ha sido suficientemente efectiva, generando brechas e inequidades. Como señala la OPECH (2006), una de las problemáticas de este periodo en Chile se refiere al debilitamiento de la educación pública, debido a crisis de calidad, inequidad y segmentación social. Al respecto indica:

Cuando surgió un alto número de escuelas y liceos privados subvencionados por el Estado, que atienden ya a más del $40 \%$ la población escolar, se agudizó la desigualdad educativa. Ello Ha significado la existencia de diferentes calidades de enseñanza en función de los estratos socioeconómicos y, más aún, en la misma educación municipal, dependiendo de si se trata de municipios ricos o pobres. (p. 4)

Como señalan López y Prado (2016), en los últimos años se han dado diversos movimientos de estudiantes secundarios y universitarios, con reivindicaciones locales y nacionales, como una respuesta a la política neoliberal aplicada en los años de la dictadura en los ámbitos económico y social y como un rechazo a los modelos políticos actuales, debido a la caída de los liderazgos políticos tradicionales, producto de una creciente desconfianza social.

Diríamos entonces que los movimientos juveniles se generan como formas de expresión del descontento social y se configuran en forma de participación en la cultura política del país. Estas organizaciones surgen como respuesta a sentimientos de disconformidad frente al sistema económico y político imperante, dado que rechazan las estructuras vigentes, sus formas de actuación y la for- 
ma en que actúa la sociedad en general ante los problemas que les acontecen. Ellos se perciben aislados, valorativa, normativa y afectivamente, del resto de la sociedad, e incluso de sí mismos. Igualmente sienten que no hay un proyecto, un futuro, una sociedad de los incluya. Por ello, "cuando manifiestan su descontento en las calles, son reprimidos, estigmatizados y criminalizados" (Sandoval, 2012, p. 60).

Si bien en 2006 comienza la llamada "revolución de los pingüinos", previamente existieron en nuestro país diversos movimientos estudiantiles que, desde 1985, se adhirieron a la lucha en contra del régimen militar instaurado en Chile, generando los primeros medios de representación, como la FESES (Federación de Estudiantes Secundarios de Santiago). De igual manera, en 2001 se presenta el llamado "mochilazo", reivindicativo ante el alza del pase escolar (Silva, 2014, p. 9; Zarzuri, 2008, p. 8).

Considerando estos ejemplos históricos, la "revolución pingüina” se presentó como movimiento que expresó públicamente el descontento juvenil generalizado de los estudiantes de secundaria; miles de estudiantes que tomaron sus colegios, hablaron fuerte y remecieron los cimientos del poder institucionalizado. Estos movimientos de los jóvenes de secundaria fueron gradualmente respaldados por otros actores: profesores, apoderados, trabajadores (Zarzuri, p. 9). Sin embargo, el gobierno y los medios de comunicación intentaron bajar el perfil al movimiento, "tratando de construir una imagen de niños chicos, infantilizándolos, de poco maduros", por tanto, ignorándoles como actores válidos (Zarzuri, p. 9); "así como la cooptación no permitió ningún cambio ni mejora sustancial en la calidad de la educación” (Sandoval, 2012, p. 53).

Este movimiento se inició con una serie de tomas de colegios (al terminar el primer semestre del año escolar), que reclamaban una mejora sustancial de las condiciones infraestructurales de los establecimientos, pero también, desde el principio, apuntaba a reformular la calidad de la enseñanza y el rol del Estado como agente 
en dicho proceso. El diagnóstico que hacían los propios estudiantes era el de una crisis aguda en la educación chilena y un colapso total en el sistema público (Institut de recherche et débat sur la gouvernance-IRG, 2007).

Durante esta etapa, las movilizaciones de los jóvenes estudiantes cambiaron la mirada hacia la educación en el país, lo cual se evidenció en las diversas reclamaciones presentadas, según recogen Gerter y Ramos (2011), entre las que destacan:

- Derogación de la Ley Orgánica Constitucional de Enseñanza.

- Derogación del Decreto 524, publicado el 11 de mayo de 1990, que regula los centros de alumnos.

- Fin de la municipalización de la enseñanza.

- Estudio y reformulación de la Jornada Escolar Completa, JEC.

- Gratuidad de la Prueba de Selección Universitaria, PSU.

- Pase escolar gratuito y unificado.

- Tarifa escolar gratuita en el transporte escolar para la Educación Media.

Para 2008 se dieron nuevas movilizaciones de estudiantes en Chile, quienes, mediante manifestaciones, protestas de descontento ciudadano, reclamaban por el incumplimiento de los acuerdos a los que se llegó en 2006 y la inminente aprobación de la Ley General de Educación (LGE).

En 2011 se presentó un nuevo movimiento estudiantil que se abanderó bajo el eslogan de cambiar el sistema educacional, potenciando la calidad por sobre el mercado. Miles de estudiantes salieron a las calles y entre los líderes estaban los entonces presidentes de la FEUC, Giorgio Jackson (Revolución Democrática); y de la FECH, Camila Vallejo (Partido Comunista), quienes actualmente son diputados de la República (Muñoz \& Bustos, 2016, s/p).

Este movimiento juvenil se ha reconocido como el más fuerte en materia de impacto social y organizacional, estableciendo demandas que no solo avalaban el desarrollo universitario como sector precursor del movimiento en este periodo, sino que de los secto- 
res escolares y secundarios de la educación chilena, principalmente la estatal, desarrollando la demanda más variada y contundente de las últimas décadas (UNICEF, 2014).

En los años posteriores se han dado movilizaciones, paros, tomas, que han buscado, entre otras reinvindicaciones, autonomía política, docente y administrativa de las universidades; cogobierno tripartito; asistencia libre e ingreso irrestricto; organización estudiantil; libertad y periodicidad de cátedra; pluralismo doctrinario; centralidad del alumno; enseñanza gratuita, laica y de excelencia; elevado presupuesto educativo (Biagini, 2012, citado en López y Prado, 2016). Otras demandas son en pro de los derechos humanos, demandas animalistas, de diversidad sexual, equidad de género, entre otras, tal y como señala Garcés (2012).

Estos movimientos fueron reprimidos por las autoridades durante las protestas masivas mediante detenciones bajo la acusación de "desorden en la vía pública" o "destrozos en la vía pública" (Zarzuri, 2008, p. 12). La mayoría de estas detenciones son de corta duración, exigiendo la presencia de los padres para liberarlos.

\section{Universidad Católica Silva Henríquez: repercusiones de un movimiento}

La Universidad Católica Silva Henríquez es una institución de educación superior perteneciente a la comunidad salesiana, instalada hace 36 años en Santiago de Chile. Su empeño y preocupación está en garantizar la calidad de su formación académica, profesional, humana y cristiana a los jóvenes. En tal sentido, busca "continuar el acompañamiento de los jóvenes en un período de su vida en que toman las decisiones más determinantes y (...) ofrecer una oportunidad de acceso a la universidad a muchos jóvenes en inferioridad de condiciones económicas y sociales" (IUS, 2003, p. 5). En tal sentido, la UCSH se declara a sí misma como una universidad privada "socialmente responsable y comprometida con los principios de equidad e inclusión en tanto Universidad, católica y Salesiana" (UCSH, 2014, p. 5). 
Según el informe de la Unidad de Planificación y Análisis institucional (UCSH, 2018), los estudiantes en la UCSH alcanzan un promedio de edad de 20 años, y más del $80 \%$ declara ser universitario por primera vez, mientras que el $70 \%$ califica como primera generación de su familia que accede a la educación terciaria. En cuanto a la condición socioeconómica, el $80 \%$ de los estudiantes pertenece a los tres primeros quintiles, por lo que más del $60 \%$ proviene de colegios particulares subvencionados y más del $30 \%$ del sector municipal. Asimismo, un $16,5 \%$ se identifica con la izquierda y un $4,8 \%$ con la derecha. La mayoría de ellos vive en comunas altamente pobladas en Santiago, como Puente Alto y la Florida; además, existe un 30\% de estudiantes que informa estudiar y trabajar, por la situación económica inestable de su grupo familiar, aunque la mayoría se siente apoyada por su familia.

En la UCSH, los movimientos estudiantiles se han manifestado en diversas oportunidades de acuerdo con las coyunturas nacionales educativas. Esto se engarza con petitorios internos referidos a mayor participación, gratuidad y aspectos de calidad de sus planes de estudio, entre otras motivaciones. A través de sus relatos, los estudiantes logran hacer inteligibles estos acontecimientos al resignificarlos (Morales, 2005). Por ello, escuchar estas voces y recoger estas experiencias reviste un gran interés investigativo.

\section{Propuesta de investigación}

Se reconoce que la vida social es interpretativa y, en tal sentido, las prácticas sociales son "significantes" y, por tanto, "organizadas socialmente, conformadas de manera histórica y mediante realizaciones informadas políticamente" (Sancho, et al. p. 1159). Por ello, la experiencia, el mundo, la vida es significativa en la medida en que es narrada, (...) y que el mundo no es independiente de las versiones que se tengan de él (Morales, 2005). Se busca entonces la comprensión, es decir, la captación de las relaciones internas y profundas mediante la penetración en la intimidad de los participantes, para ser entendida desde adentro, en su novedad, respetando la originalidad y la indivisibilidad de los 
fenómenos; en lugar de parcelar lo real, la comprensión respeta su totalidad vivida.

La perspectiva teórica de este estudio es interpretativa hermenéutica, porque se busca comprender e interpretar la realidad construida por los diversos participantes, reconociendo los elementos culturales e históricos subyacentes. Los fenómenos que se indagan son dinámicos, holísticos, están interconectados (Sandín, 2004). No obstante, a través de una hermenéutica de validación es posible capturar los significados de los textos (observaciones, entrevistas) y los sentidos atribuidos.

En cuanto al tipo de diseño, se empleó el enfoque biográfico narrativo, que resulta más apropiado para abordar la construcción simbólica de los movimientos sociales. Ello en tanto las creencias y explicaciones que se asignan a las cosas o eventos tienen una organización narrativa o en tramas, como indica Bruner (1990). En tal sentido, afirma," hay un actor llevando a cabo una acción, con una meta, en un escenario, a partir de unos instrumentos" (p. 83).

La estrategia metodológica fue el desarrollo de grupos de discusión, técnica cualitativa de tipo analítica para el estudio de los contenidos latentes en las opiniones sociales, la que consiste en reunir un grupo de personas que comparten opiniones acerca de un tema determinado, bajo la guía de un coordinador, quien interviene de forma no directiva. El objetivo es reconocer la estructura de sentido compartida por los integrantes.

\section{Resultados}

La muestra estuvo constituida por 38 estudiantes y egresados de la UCSH que han sido representantes estudiantiles de la federación de estudiantes o delegados de carrera de los últimos 10 años (cohortes, 2006-2016). Es decir, son jóvenes estudiantes de la UCSH que estuvieron presentes en algún tipo de manifestación, como asambleas, paros, marchas, tomas, grafitis, entre otros. Las respuestas se analizaron cuidadosa y profusamente, en reiteradas lecturas de la información, con el fin de identificar 
conceptos, categorías y expresiones que dieran cuenta del tema de estudio. Es decir, se trató de analizar el corpus en unidades, para proceder, posteriormente, a su agrupamiento en categorías o subcategorías, siguiendo el criterio de analogía. Por tanto, se pasó por los diferentes niveles de procesamiento de la información, abstracción, síntesis, conceptualización, contrastación teórica y deducción.

A continuación, se dará respuesta a los objetivos que orientaron el proceso investigativo $y$, posteriormente, a los hallazgos emergentes, teniendo como base la sistematización de las narrativas aportadas por estudiantes y egresados de la UCSH que han vivido los movimientos estudiantiles de los últimos 10 años. A partir del año 90 en el país hubo una transición democrática posdictadura; sin embargo, esta no se gestionó de manera participativa ni efectiva, como tampoco fue representativa de los diferentes sectores, espacios e intereses, por ello, no convocó a la participación plena. Se percibe que el sistema democrático actual ha velado por los intereses de unos pocos. Este sistema debería ser más bien una gestión por los derechos e intereses comunes, como por ejemplo el de la libre expresión social. Como señala Zerán (2017), "en Chile de fines del 2016 e inicio del 2017. (...), pese a las demandas de cambio y de nuevas voces, se observa crisis de la política, de abstención de protagonismo de los movimientos sociales, todo parece estar convulsionado" (p. 15). Respecto de lo anterior, los líderes actualmente buscan direccionar a sus seguidores más que ser sus representantes, imponiendo así, en cierta medida, su voluntad. Proponen una democracia inclusiva, con mayor enfoque social, basada en deberes y derechos, responsable y respetuosa por el bien común de todos; que escuche a las personas como medio de llegar hacia el colectivo, en algunos casos a proponer una dictadura del proletariado o una utopía anárquica. Respecto de este punto un joven señala,

Para mí la democracia es una responsabilidad conjunta en la que todos los sectores de la sociedad tienen derechos y deberes, principalmente el derecho de ser escuchados y participar, pero 
también el deber de escuchar al otro en una instancia participativa conjunta (...). (E. 9)

Consideran igualmente que la democracia se ha corrompido, debido a las inequidades producidas por el modelo neoliberal, así como por las acciones contaminadas e individualistas de los partidos tradicionales. En este sentido, invitan a visualizar y analizar de manera más exhaustiva y consciente la orientación e intencionalidades de los agentes políticos, pues no se ha conformado un sistema democrático que los represente y por ello, algunos no desean participar a través del voto. Al respecto, otro entrevistado indica,

(...) Hoy en día tenemos una estructura política muy mal diseñada, respecto a eso no hemos podido erradicar ciertas prácticas que quedaron de la dictadura (...). (E. 11)

Desde esa perspectiva de democracia, los entrevistados se identifican de igual manera como personas políticas, no solo ni necesariamente en lo electoral, sino como sujetos participes en la construcción social del país, por ello se involucran activamente en estos movimientos juveniles en los cuales pueden expresar sus sentimientos y emociones. Ejemplo de ello,

(...) Yo me considero un sujeto político, tanto en mi participación en distintos ámbitos de la sociedad chilena, como también en la forma que me expreso en mi diario vivir (...). (E. 10),

Respecto de la participación democrática, algunos estudiantes consideran que la mayoría de sus compañeros no actúa ni participa como sujetos políticos, no votan en las elecciones, debido a un sentimiento de agotamiento de las formas tradicionales de participar, estas instancias no convocan porque el diálogo está cerrado. Debido a ello, las formas tradicionales de convocarlos no los invitan a ser partícipes.

(...) te puedo decir que la juventud está muy perdida, políticamente hablando yo creo que el neo-hippismo se los consumió 
(...) se despolitizaron completamente después del actuar de los funcionarios en el 2014 (...). (E .6)

Desde este punto de vista, consideran que la Universidad se mantiene a distancia y no promueve la construcción de sujetos políticos en los distintos espacios que componen la institución; más bien, los jóvenes se sienten reprimidos, o vistos como problemáticos por las autoridades, quienes tienen una visión diferente de su accionar. Ejemplo de esto, es un testimonio como el siguiente,

(...) incluso aquí en la Universidad cuando vemos que hay espacios despolitizados es porque nuestros mismos compañeros se aburrieron de hacer política (...). (E.11)

De igual manera, los participantes indican que se están preparando para ser representantes o líderes sociales y políticos mediante una formación de base, ya sea en su entorno familiar o mediante la asistencia a eventos de liderazgo juvenil (nacionales e internacionales). Esta formación es orientada por asociaciones independientes y partidos políticos generalmente de izquierda, los cuales buscan fortalecer roles de liderazgo. Ejemplo de esto son las actuales figuras parlamentarias y ex representantes del movimiento estudiantil, como Camila Vallejo o el "ex comandante conejo", que estudió en la UCSH. Refrenda esta idea el siguiente relato:

(Refiriéndose a los partidos políticos) (...) hay jóvenes que piensan que son instancia validas quizás no la única, pero una de muchas opciones (...) la política institucional también es válida, hay otras formas que no se descartan (...). (E. 9)

Por ello, los entrevistados consideran que desarrollan nuevas formas de hacer y actuar políticamente. De esta manera, creen que la política se hace en el día a día, como por ejemplo participar en una instancia como este estudio es considerada una forma de hacer política, dado que intercambian opiniones y se construyen nuevas realidades. $\mathrm{Al}$ respecto dicen: 
(...) entonces deconstruir esa realidad por ejemplo es hacer política, hay que empezar a prestar oído a las distintas formas y lugares de hacer política en base a las relaciones sociales (...). (E. 11)

En cuanto a la capacidad de influencia de los entrevistados como miembros de los movimientos juveniles, ellos creen que poseen la formación y las capacidades necesarias para actuar y producir el cambio social esperado, dada su posesión de un sistema de valores, tales como, el saber escuchar, la empatía, la comunicación fluida, la transparencia, entre otras. Con base a lo anterior, se han involucrado en distintas actividades institucionales, así como lo hicieron en el liceo, para ser voceros y ayudar a sus compañeros a generar los cambios requeridos. Ejemplo de ello,

(...) pero siento que el movimiento fue mutando y se transformó en la voz de la sociedad en tanto al descontento social, pero también en levantar propuestas; entonces, muchos sectores de la sociedad que no están en la universidad o en las escuelas, se dieron cuenta de que el movimiento representaba esa voz que muchas veces o por mucho tiempo estuvo callada, y a sea por la dictadura o porque la sociedad no se estuvo comprometiendo con la participación política; por lo tanto el movimiento estudiantil tiene un corte político (...). (E. 8)

Con base en estas narrativas de los estudiantes y egresados de la UCSH se puede afirmar que los entrevistados se reconocen como agentes políticos capaces de movilizar personas, ideas y proyectos de cambio en torno a la educación y la transformación del país. Ellos comprenden y actúan en lo político, denunciando y demandando cambios estructurales; para esto, utilizan estrategias que emplazan a la institución y a la sociedad civil a visibilizar una problemática postergada y la instalan en la agenda pública. Estas voces jóvenes se han organizado para ser escuchados y respaldados por la sociedad, la que apoya explícitamente estas demandas (calidad de educación, modificación del CAE, gratuidad). Por ello, a través de sus manifestaciones críticas y algunas veces disruptivas, 
gradualmente obtienen respuestas parciales del gobierno y sus estamentos para atenuar el conflicto.

En su gestión, el movimiento estudiantil se construye con base en procesos de participación y discusión democráticos, teniendo como fin la búsqueda o establecimiento de un objetivo común que satisfaga las distintas necesidades expresadas por la mayoría de los entrevistados. Lo anterior se relaciona preferentemente con el ámbito educativo, es decir, acercando al joven al sistema organizativo del Estado y, con ello, a la política, tomando distancia de la visión clásica de la política partidista.

(...) democratizar los espacios, es decir, mejorar la participación en la toma de decisiones y que esto no esté solo en el consejo universitario o que esté en mano de 4 o 5 personas. (E. 8)

Por otra parte, las instancias más clásicas empleadas para la toma de decisiones son la asamblea, las votaciones a mano alzada o por urna, como también reuniones estructuradas entre estudiantes, funcionarios y o autoridades. Cabe destacar que dentro de estas instancias existen sujetos o grupos encargados de mantener el quorum, y la organización y el respeto entre los estudiantes desde allí se configuran entidades como las federaciones, las que están compuestas por estudiantes electos democráticamente por la mayoría, los que lideran el proceso.

(...) una de las características que debe tener un líder, es que tiene que saber escuchar no solo a quienes tiene a su lado sino que al colectivo general: saber mover masas a partir de eso (...). (E. 3, S. 2)

En este proceso participativo la comunicación es un factor relevante, dado que a través de ésta se expresan y recogen las preocupaciones generales y demandas del movimiento.

(...) existe igual una cierta disposición al dialogo, es una universidad de puertas abiertas, la posibilidad de hablar con las autoridades, el acceso que tenemos a hablar con los directivos, con profesores, existe, es real, pero es ganado. (E.1, S. 3) 
A continuación se darán a conocer las diversas acciones y prácticas que los participantes realizan apelando a su desarrollo del ejercicio ciudadano y político.

Primeramente, se destaca que el movimiento estudiantil ha estado marcado por una serie de prácticas y acciones, las cuales son dotadas por los mismos participantes de un así llamado "carácter icónico o histórico", basado en su efectividad como medio de visualización de varias problemáticas, como también en la gran convocatoria que gestiona para el desarrollo de un movimiento social, siendo los principales medios las marchas, paros y, en casos extremos, ante la negativa de la autoridad, la toma (ocupación ilegal). Unas de las expresiones de presión y de denuncia han sido las marchas, principalmente la de 2006, 2010-2012, 2014, en las cuales se demandaba al Estado una educación de calidad y gratuita, entendiendo que existía una barrera que negaba a los jóvenes acceder a la educación superior. Estas demandas exceden a las situaciones internas de la UCSH.

(...) la marcha en un momento fue importante porque visibilizó la demanda de los estudiantes y permitió que la sociedad se movilizara; además los trabajadores históricamente se han apropiado de la marcha para dar a conocer sus problemas (...). (E. 8)

Las prácticas y acciones por medio de las cuales se da a conocer el descontento de los estudiantes también han evolucionado. Si bien éstos han mantenido ciertas prácticas y formas de manifestación históricamente efectivas, como las marchas, paros y tomas, estas han sido apropiadas con nuevos tintes, como un medio de llamar a la participación masiva, desde un punto de vista más creativo y didáctico, en donde se pongan en práctica y desarrollen las habilidades personales y académicas de los participantes. Estos grupos se estructuran en cuatro momentos: la discusión, la toma, el desalojo, la posible retoma. Esto aplica de manera parecida con el paro. En el caso de la asamblea, se estructura en organización, difusión, realización, reconvocación desde una perspectiva construccionista en las ciencias sociales. 
(...) surgieron buenas iniciativas de acción como talleres de feminismo, de participación política y social, principalmente voluntariados que son cosas que nos ayudan a romper nuestras lógicas (...). (E. 11, S. 1)

Con base a lo anterior, los estudiantes realizan diversas prácticas, algunas de las cuales son aceptadas y validadas y concordadas con otros actores sociales, académicos, familias, mientras que otras expresiones son rechazadas y cuestionadas por su contenido material y simbólico contestatario y violento, que en ocasiones atenta y desdibuja el propósito inicial de denuncia. Los estudiantes no desconocen ni justifican como válidos los incidentes que terminan tergiversando la acción masiva y los objetivos del movimiento estudiantil.

(...) la marcha es una herramienta válida, pero no es la única que existe y es una tarea que va quedando actualmente para los movimientos a nivel global en la sociedad, como también el cómo incidir en otros espacios y comunidades que no han participado (...). (E. 11, S. 1)

Por tanto, los estudiantes desarrollan posturas críticas, de acción y concientización en contra de las prácticas violentas que se presentan en el movimiento, rechazándolas por completo y señalando que son una minoría. Desde este punto, gestionan medios para salvaguardar los principios y objetivos del movimiento, como también para promover la conciencia colectiva e integridad de los participantes y de los espacios públicos y privados.

(...) dentro de las conversaciones que tenía con mis compañeras que se quedaban era que había peleas entre personas que solo venían a destruir, entonces yo pensaba “Dónde está el anarquismo si estás igual dentro de un sistema?" es como tonto, pero eso ayudó a que se hiciera un filtro de ingreso a la toma (...). (E. 5)

En estos movimientos podemos identificar algunos fenómenos de masa que alteran una marcha: una provocación, tirar piedras, insultar, agredir, desviando el objeto de rabia, molestia o resenti- 
miento a un objeto que represente para ellos el Estado o el agente opresor. Como por ejemplo semáforos, carabineros, paredes, recintos comerciales o privados, arboles, locomoción colectiva, el Metro, entre otros.

\section{Reflexiones finales}

El resultado más evidente encontrado en el estudio es la relevancia que han tenido los diversos movimientos juveniles en los procesos de ajuste del sistema educativo, tanto del país como de la institución. Se puede mencionar, que efectivamente, en los relatos se manifiesta el descontento y desencanto frente a la estructura, a los procesos políticos, a las prácticas, a las formas de operar en el sistema parlamentario y el Estado.

Específicamente, piensan que todavía quedan resabios de la dictadura; mencionan la Constitución, la democracia representativa, el modelo centralista, la toma de decisiones, los tiempos de respuesta (demorados) y la profundidad de los cambios y reformas establecidas frente a las requeridas por la sociedad civil. En este sentido, los entrevistados señalan que las actuaciones gubernamentales han estado teñidas o son poco transparentes, porque perciben que existe corrupción, altos sueldos a los parlamentarios y colusión en los contratos o en los beneficios con los empresarios. Este hallazgo, también ha sido señalado en el estudio del Programa de las Naciones Unidas para el Desarrollo (PNUD, 2003), quienes encuentran que solo un $40 \%$ de los jóvenes chilenos respalda la democracia, y que "existe una serie de cifras y análisis que demuestran un creciente distanciamiento entre el mundo juvenil y la política" (p. 16); aspecto ratificado por Sandoval (2012), cuando indica que "la postura juvenil frente al mundo institucional es de distancia, de desimplicación, de rechazo" (p. 55).

Es decir, se percibe una distancia entre los movimientos juveniles en Chile y los partidos políticos. Como señala Garcés (2012), "se configura un cuadro social y político con dos componentes; el 
movimiento social por un lado y por el otro una crisis de credibilidad y legitimidad hacia el Estado". Algunos entrevistados consideran que sus propios líderes universitarios, al obtener cargos en el parlamento y en los ministerios, se trasformaron en oficialistas, olvidando su origen y las preocupaciones de base por las que luchaban en un momento. Lo anterior se evidencia en las medidas paliativas o "de parche" que no transforman los problemas de base; ejemplo de ello, es la no realización de las reformas históricas demandadas, como la condonación del CAE, ranking, PSU, gratuidad universal, lucro, entre otros. Se cuestiona también que estas personas asumieron rápidamente los mecanismos ya establecidos, acoplándose al modelo imperante. Este distanciamiento es más claro en el caso de partidos como el socialista y el demócrata cristiano (...) y el Partido Comunista (Garcés, 2012, p. 335).

Se observa en las narrativas una percepción idealizada de la sociedad y el Estado, en el cual las medidas demandadas deberían establecerse en forma inmediata, porque todo es posible, sin necesidad de concertación o negociación. Mientras que los jóvenes que avanzan en su trayectoria académica desarrollan un discurso más fundamentado y con mayores elementos de análisis, que les permite poner en perspectiva la realidad del país. Algunos consideran que la baja votación presidencial se debe a la falta de credibilidad y de formación política del estudiantado, provocando reacciones que conducen a distintos conflictos y problemáticas dentro del movimiento.

Frente al supuesto de que existen nuevas formas de representación de la democracia y del rol ciudadano, se encuentra que ellos promueven formas de aproximarse a la política que son distintas, siendo relevantes las estrategias comunicativas que emplean antes de generar los petitorios, las que reflejan el malestar de la comunidad estudiantil, tanto a nivel institucional como nacional. Como se ha mencionado, los movimientos tienen modos de operar propios para manifestar sus descontentos internos y dar señales al resto de la comunidad que hay una situación que atender y solucionar. 
Otra forma de operar que se percibe en los movimientos es el surgimiento de los colectivos, que emergen de acuerdo con las necesidades y temáticas prioritarias o que se instalan en la agenda, los que le proporcionan mayor cohesión. Lo anterior, a modo de denunciar y visibilizar temáticas que requieren procesos de equidad, tales como la equidad de género, entre otras. En las narrativas, se observa que a las directivas de la Universidad se las percibe como el enemigo, o el otro, el malo, el que destruye las ilusiones. Por ello, tienen dificultades para sentarse posteriormente con ellos a negociar y llegan a realizar requerimientos de cambio sin grandes argumentos que los sustenten. Por otra parte, los estudiantes que asumen el rol de representantes tienen un imaginario mesiánico de sí mismos, se consideran los salvadores, capaces de llevar a cabo grandes trasformaciones. Reflejo de ello son las distintas manifestaciones de carácter masivo que se dan frente a situaciones que afectan el bienestar y la calidad de vida de los ciudadanos, algunas de las cuales ostentan alegorías y sátiras tanto novedosas como creativas, que, poco a poco, fueron incentivando a los distintos grupos de la sociedad chilena. Esto ha estimulado a los jóvenes a apropiarse de distintas causas sociales y a manifestarse contra estructuras dejadas como resabio de la dictadura militar, época durante la cual las diversas manifestaciones masivas fueron vistas de manera problemática o señaladas como características de la rebeldía juvenil. Los movimientos entonces han logrado legitimarse como un medio de reivindicación del descontento social. Como señala Reguillo (2000):

El siglo XXI arranca con evidentes muestras de una crisis politico-social. De maneras diversas y desiguales, los jóvenes han seguido haciendo estallar las certezas y han continuado señalando, a través de los múltiples modos en que se hacen presentes, que el proyecto social privilegiado por la modernidad en América Latina ha sido, hasta hoy, incapaz de realizar las promesas de un futuro incluyente, justo $y$, sobre todo, posible. (p. 22) 
Los jóvenes de la Universidad Católica Silva Henríquez (UCSH) como sujetos políticos en los movimientos sociales/Brito et al.

\section{Referencias bibliográficas}

Bendit, R. (2000). Adolescencia y participación: Una visión panorámica en los países de la Unión Europea. Anuario de psicología, 31(2). Facultad de psicología. Universidad de Barcelona. Recuperado de http://www.raco.cat/index.php/anuariopsicologia/article/viewFile/61543/88398

Braslavsky, C. (2006). Diez factores para una educación de calidad para todos en el siglo XXI. REICE, 4(1). Recuperado de http:// www.rinace.net/arts/vol4num2e/art5_htm.htm

Garcés, M. (2012). El despertar de la sociedad. Los movimientos sociales en América Latina y Chile. Santiago de Chile: LOM ediciones.

García-Cabrero, B., Loredo, J., Luna, E. y Rueda, M. (2008). Modelo de evaluación de competencias docentes para la educación media y superior. Revista Iberoamericana de Evaluación Educativa, 1(3), 124-136.

Gerter, D. y Ramos, C. (2011). Movimiento Estudiantil en Chile: Una respuesta a la carencia de Estado. Valdivia: Universidad Austral de Chile.

Institut de recherche et débat sur la gouvernance-IRG. (2007). El movimiento estudiantil en Chile, o la marcha de "los pingüinos". Recuperado de: http://www.institut-gouvernance.org/es/ analyse/fiche-analyse-348.html\#h1

IUS. (2003). Identidad de las Instituciones Salesianas de Educación Superior (IUS). Roma: Direzione Generale Opere Don Bosco.

Laurin-Fenette. (1976). Las teorías funcionalistas de las clases sociales: sociología e ideología burguesa. Bogotá: Siglo XXI. Recuperado de https://books.google.cl/books?isbn=8432300713

Lind, G. (2003). Psicología de la Moral y Democracia, y Educación. Universidad de Konstanza. Recuperado de http://www.unikonstanz.de/ag-moral/about-sp.htm[obtenida el 10 Oct 2004]

López, D. y Prado, M. (2016). ¿Qué deseaban los estudiantes universitarios chilenos el año 2011? Altre Modernità, [S.1.], 218-233. DOI: http://dx.doi.org/10.13130/2035-7680/7064

Mena, A. y Méndez, J. (2009) La técnica de grupo de discusión en la investigación cualitativa. Aportaciones para el análisis de los procesos de interacción. Revista Iberoamericana de Educación OEI, 49(3). Recuperado de https://rieoei.org/RIE/ article/view/2094 
Morales, J. (2005). Teoría narrativa de la psicología asocial en el modo de ser literario. Tesis doctoral. Departamento de Psicología social. Universidad Autónoma de Barcelona.

Muñoz, C. (2002) Psicología científica o psicología popular: Un modelo narrativo de la mente. A Parte Rei. Revista de filosofía, 24. Recuperado de http://serbal.pntic.mec.es/ cmunoz11/ pspopular.pdf

Muñoz, D. y Bustos, M. (2016). ¿Quépasa con el movimiento estudiantil en el año decisivo de la Reforma? La Tercera, 10 abril. Recuperado de http://www.latercera.com/.../680-675914-9-quepasa-con-el-movimiento-estudiantil-en-el-a.

OPECH. (2006). La crisis del sistema educativo chileno. Diagnóstico y propuestas. Recuperado de http://www.opech.cl/movisociales/propuestas/doc_colegio_profes.pdf

Programa de las Naciones Unidas para el Desarrollo - PNUD. (2003). Transformaciones culturales e identidad juvenil en Chile. Temas de desarrollo humano sustentable, 14. Recuperado de http://www.pnud.cl.

Reguillo, R. (2000). Emergencia de culturas juveniles. Estrategias de desencanto. Enciclopedia latinoamericana de sociocultura y educación. Buenos Aires: Norma.

Rodríguez, E. (2013). Movimientos juveniles en América Latina: entre la tradición y la innovación. Texto preparado para su presentación en la IX Reunión del Foro de Ministros de Desarrollo Social de América Latina (Tegucigalpa, Honduras, 6 y 7 de marzo de 2013). Recuperado de http://www.celaju. net/wp-content/publicaciones/2014/05/Movimientos-Juveniles-ALC.pdf

Sancho, J., Hernández, F., Herraiz, F. y Vidiella, J. (2009). Una investigación narrativa en torno al aprendizaje de las masculinidades en la escuela. RMIE, octubre-diciembre, 14(43), 1155-1189

Sandoval, M. (2012). La desconfianza de los jóvenes: sustrato del malestar social. Última década, 20(36), 43-70. Recuperado de https://dx.doi.org/10.4067/S0718-22362012000100003

Silva, P. (2014). La "Revolución Pingüino" y el cambio cultural en Chile. Archivo de Chile.

UCSH. (2018). Caracterización de estudiantes de Primer año 2018. Santiago de Chile: Universidad Católica Silva Henríquez.

UNICEF. (2014). La voz del movimiento estudiantil 2011. Educación pública, gratuita y de calidad. Santiago de Chile: Ed. Miguel Lafferte y Carolina Silva Gallinato E.I.R.L. 
Los jóvenes de la Universidad Católica Silva Henríquez (UCSH) como sujetos políticos en los movimientos sociales / Brito et al.

Velasco, M. (2003). La adolescencia en el proceso del desarrollo humano; perspectiva constructivista. Ciudad redonda. Recuperado de http://www.ciudadredonda.org/caminos/laicos.htm

Weber, M. (1996). Economía y sociedad. México: Fondo de Cultura Económica, p. 18.

Zarzuri, R. (2008). Algunos ejes interpretativos sobre los movimientos sociales y la represión de la protesta social en Chile. Santiago de Chile: Centro de Estudio Socioculturales CESC.

Zerán, F. (2017). Repensar la izquierda, en Chile actual: Crisis y debate desde las izquierdas (pp. 9-16). Santiago de Chile: Lom Ediciones. 